\title{
METODOLOGIA PARA PREVISÃO DA EFICIÊNCIA DE DESFOSFORAÇÃO DE FERRO GUSA UTILIZANDO TERMODINÂMICA COMPUTACIONAL*
}

\author{
Heitor Cristo Clem de Oliveira ${ }^{1}$ \\ Silas Gambarine Soares ${ }^{1}$ \\ Felipe Costa Broseghini ${ }^{1}$ \\ Sábata Marla Reis Durães Oliveira ${ }^{1}$ \\ José Roberto de Oliveira ${ }^{2}$
}

\section{Resumo}

Devido às influências deletérias do fósforo sobre as propriedades do aço e os teores cada vez mais altos de fósforo no minério, a remoção do mesmo através do processo de desfosforação do ferro-gusa ganha cada vez mais importância. Este trabalho tem como objetivo a avaliação termodinâmica de misturas a base de CaO$\mathrm{FeO}$ utilizadas no processo de desfosforação de ferro-gusa. A avaliação termodinâmica foi realizada através dos softwares termodinâmicos, ThermoCalc e FactSage, os quais mostraram-se eficazes na determinação da concentração de fósforo de equilíbrio no metal, fases presentes e temperatura de fusão das misturas. Palavras-chave: Pré-tratamento de ferro-gusa; Desfosforação; Termodinâmica computacional.

\section{METHODOLOGY FOR DEPHOSPORIZATION EFFICIENCY PREDICTION OF HOT METAL USING THERMODYNAMICS SOFTWARES}

\section{Abstract}

Due to the injurious influences of phosphorus on the properties of steel and increasingly high contents of phosphorus in the ore, the removal of it through the process of hot metal dephosphorization has achieved an increasing importance. The objective of this article is the thermodynamic evaluation of mixtures based on $\mathrm{CaO}$ $\mathrm{FeO}$, used in the process of hot metal dephosphorization. The thermodynamic evaluation was performed using computational thermodynamic software FactSage, which were effective in determining the equilibrium phosphorus concentration in the metal, present phases, and melting temperature of the mixtures.

Keywords: Hot metal; Pre-treatment; Dephosphorization; Computational thermodynamics.

1 Engenheiro Metalurgista e Mestrando em Engenharia Metalúrgica e de Materiais, IFES, Vitória, ES, Brasil.

2 Prof. Dr., Instituto Federal do Espírito Santo (IFES), cursos de graduação e pós-graduação em Engenharia Metalúrgica e de Materiais, IFES, Vitória, ES, Brasil. 


\section{INTRODUÇÃO}

A presença de fósforo no metal afeta adversamente algumas propriedades do aço como a tenacidade e a resistência. Além disso, a presença de fósforo causa a segregação intergranular durante o lingotamento contínuo e deteriora a ductilidade e resistência de produtos como tubos, aços criogênicos e placas de vasos de pressão [1]. Portanto, torna-se necessário a remoção do fósforo a fim de obter aços com maior limpidez e qualidade. O fósforo pode ser removido mediante processos redutores ou oxidantes, sendo este último o processo mais aplicado devido ao baixo custo do $\mathrm{CaO}$ e de conseguir ambientes oxidantes através da injeção de $\mathrm{FeO}$ [2].

A etapa de desfosforação de ferro-gusa pode ser realizada no carro torpedo, na panela de transferência de gusa ou até mesmo em um convertedor específico para este fim. A escolha do equipamento destinado a esta operação depende do layout e da limitação de espaço da empresa, bem como da relação custo-benefício de se implantar tal processo. Além disso, geralmente a reação é promovida através da injeção de uma mistura desfosforante à base de $\mathrm{CaO}$ e $\mathrm{FeO}$, utilizando gás nitrogênio como gás de agitação e em alguns processos é realizado sopro de oxigênio pelo topo ou pelo fundo do equipamento.

Turkdogan [3] e Heally [4] mostraram que a oxidação do fósforo pode ocorrer mediante a reação entre o fósforo dissolvido no metal e o oxigênio fornecido pelo óxido de ferro presente na escória. O pentóxido de fósforo formado é fixado na escória através do $\mathrm{CaO}$ presente na mistura, formando o fosfato tricálcio $\left(3 \mathrm{CaO} \cdot \mathrm{P}_{2} \mathrm{O}_{5}\right)$.

$$
\begin{gathered}
2 \underline{P}+5(\mathrm{FeO})+3(\mathrm{CaO})=5 \underline{\mathrm{Fe}}+\left(3 \mathrm{CaO} \cdot \mathrm{P}_{2} \mathrm{O}_{5}\right) \\
\Delta G^{\circ}=-204450+83,55 . \mathrm{T}(\mathrm{J} / \mathrm{mol})
\end{gathered}
$$

A partir da energia livre padrão, pode-se observar que a reação é favorecida por baixas temperaturas, fato esse que influencia positivamente a desfosforação do ferro-gusa, uma vez que o mesmo apresenta temperaturas baixas com relação ao aço. A avaliação termodinâmica da eficiência de desfosforação de uma mistura desfosforante é feita mediante os seguintes parâmetros: basicidade ótica $(\Lambda)$, capacidade de fosfato $\left(\mathrm{C}_{\mathrm{P}}\right)$, coeficiente de partição $\left(\mathrm{L}_{\mathrm{P}}\right)$ e coeficiente de atividade do $\mathrm{P}_{2} \mathrm{O}_{5}\left(\gamma_{\mathrm{P}_{2} \mathrm{O}_{5}}\right)$. Entretanto, no presente trabalho serão utilizados os softwares termodinâmicos ThermoCalc e FactSage na avaliação das misturas desfosforantes propostas, uma vez que tais softwares atuam na identificação tanto de variáveis termodinâmicas quanto cinéticas que afetam a reação de desfosforação.

Segundo Campos [2], a desfosforação do ferro-gusa não atinge o equilíbrio devido a fatores cinéticos associados a reação, ou seja, é necessário o transporte de massa do fósforo dissolvido no metal até a interface metal-escória (região onde a oxidação do fósforo ocorre de maneira efetiva), reagindo com o oxigênio fornecido pelas fontes de oxigênio presentes na mistura desfosforante e formando o pentóxido de fósforo $\left(\mathrm{P}_{2} \mathrm{O}_{5}\right)$. $\mathrm{O}_{2} \mathrm{O}_{5}$ formado na escória tem que ser fixado na mesma, pois caso não seja, pode ocorrer o fenômeno de reversão do fósforo a partir da escória para o banho metálico. A fixação do $\mathrm{P}_{2} \mathrm{O}_{5}$ é obtida mediante reação com o $\mathrm{CaO}$ presente na mistura, o qual forma o fosfato tricálcio $\left(3 \mathrm{CaO} \cdot \mathrm{P}_{2} \mathrm{O}_{5}\right)$. A equação geral de estudo da velocidade da reação de desfosforação é mostrada a seguir [5].

$$
-\frac{d[\% P]}{d t}=\frac{A \rho_{m}}{W_{m}} \cdot \frac{k_{m} k_{S} \rho_{S} L_{P}}{\left(k_{m} \rho_{m}+k_{S} \rho_{S} L_{P}\right)} \cdot\left([\% P]-\frac{(\% P)}{L_{P}}\right)
$$


Onde:

$k$ : Coeficiente de transferência de massa do fósforo $(\mathrm{m} / \mathrm{s})$;

$m, s$ : Denotam a fase metal e escória, respectivamente;

$\rho:$ Densidade $\left(\mathrm{kg} / \mathrm{m}^{3}\right)$;

$A$ : Área interfacial $\left(\mathrm{m}^{2}\right)$;

$W_{m}=$ Massa de metal $(\mathrm{kg})$.

O termo $\left(\frac{k_{m} k_{S} \rho_{S} L_{P}}{\left(k_{m} \rho_{m}+k_{S} \rho_{S} L_{P}\right)}\right)$ na Equação 3 é chamado de coeficiente de transferência de massa global $\left(k^{\prime}\right)$. Assim, a Equação 3 se reduz a:

$$
-\frac{d[\% P]}{d t}=k .\left([\% P]-\left[\% P_{e q}\right]\right)
$$

A Equação 4 é conhecida como a equação geral da reação de desfosforação quando o transporte de massa no metal controla a reação. No entanto, se considerarmos que a desfosforação é controlada pelo transporte de massa na escória, a equação será:

$$
-\frac{d[\% P]}{d t}=k .\left\{\left(\% P_{e q}\right)-(\% P)\right\}
$$

A partir da integração da Equação 4, é possível obter a relação para o cálculo do coeficiente de transporte de massa global do fósforo no metal (k), a qual é mostrada abaixo.

$$
\ln \left(\frac{\left[\% P_{0}\right]-\left[\% P_{e q}\right]}{\left[\% P_{t}\right]-\left[\% P_{e q}\right]}\right)=k . t
$$

Onde,

[\% $\left.\mathrm{P}_{0}\right]$ : Concentração de fósforo inicial no metal;

$\left[\% \mathrm{P}_{\text {eq }}\right]$ : Concentração de fósforo de equilíbrio no metal;

$\left[\% \mathrm{P}_{\mathrm{t}}\right]$ : Concentração de fósforo no tempo "t" no metal.

Portanto, este trabalho visa a avaliação termodinâmica da desfosforação do ferrogusa mediante utilização de mistura desfosforantes a base de $\mathrm{CaO}-\mathrm{FeO}$.

\section{MATERIAIS E MÉTODOS}

Para a realização do presente trabalho foram realizadas as seguintes etapas:

- Realização dos testes experimentais;

- Avaliação das misturas através dos softwares de termodinâmica computacional.

\subsection{Procedimento Experimental}

Inicialmente, um quilograma de ferro-gusa sólido foi carregado em cadinhos de $\mathrm{MgO}-\mathrm{C}$ e inserido em um forno de resistência elétrica MAITEC, modelo 1700-FEE a $1400^{\circ} \mathrm{C}$. Além disso, gás argônio foi soprado sobre o ferro-gusa a uma taxa de 10 $\mathrm{NI} / \mathrm{min}$, a fim de inertizar o ambiente e evitar a oxidação do metal.

Depois de aproximadamente 40 min., o ferro-gusa estava totalmente fundido. Então, foi retirada uma amostra inicial do metal através de um amostrador à vácuo e, em seguida, a mistura foi adicionada sobre o ferro-gusa através de um tubo de aço inoxidável. Depois de adicionada a mistura, um impeler de quatro pás alumina fixo a uma haste também de alumina foi inserido no banho a uma rotação de $500 \mathrm{rpm}$. 
Amostras foram retiradas nos tempos de 0,5, 10, 15, 20 e 30 minutos. A Figura 1 mostra um diagrama esquemático dos experimentos realizados.

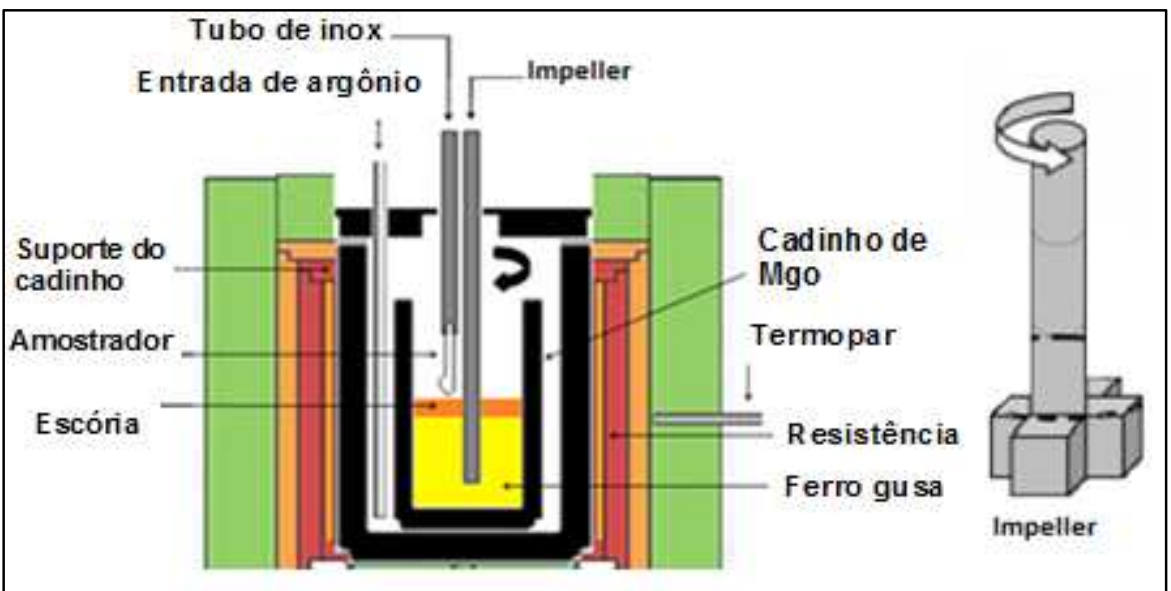

Figura 1. Diagrama esquemático dos experimentos realizados.

A Tabela 1 mostra a composição química inicial e a massa do ferro-gusa utilizada nos testes. Já a Tabela 2 mostra a composição química inicial e massa das misturas propostas e aplicadas nos testes experimentais.

Tabela 1. Composição química média do ferro-gusa.

\begin{tabular}{|c|c|c|c|c|c|c|}
\hline \multicolumn{6}{|c|}{$\%$ em massa } & \multirow{2}{*}{ Total (\%) } \\
\hline $\mathbf{F e}$ & $\mathbf{S}$ & C & Si & $\mathbf{P}$ & Mn & \\
\hline 94,66 & 0,005 & 4,45 & 0,32 & 0,12 & 0,39 & 100 \\
\hline
\end{tabular}

Tabela 2. Composição química inicial e massa das misturas desfosforantes.

\begin{tabular}{|c|c|c|c|c|c|c|c|c|}
\hline \multirow{2}{*}{ Mistura } & \multicolumn{6}{|c|}{ Composição (\%) } & \multirow{2}{*}{$\begin{array}{l}\text { Relação } \\
\mathrm{CaO} / \mathrm{FeO}\end{array}$} & \multirow{2}{*}{$\begin{array}{l}\text { Massa da } \\
\text { Mistura (g) }\end{array}$} \\
\hline & $\mathrm{FeO}$ & $\overline{\mathrm{CaO}}$ & $\mathrm{SiO}_{2}$ & $\mathrm{Al}_{2} \mathrm{O}_{3}$ & $\mathrm{MgO}$ & MnO & & \\
\hline MD1 & 71,6 & 25,6 & 1,65 & 0,68 & 0,13 & 0,34 & 0,36 & 65,0 \\
\hline MD2 & 65,8 & 31,5 & 1,60 & 0,64 & 0,15 & 0,31 & 0,4 & 65,0 \\
\hline MD3 & 60,0 & 37,4 & 1,51 & 0,63 & 0,18 & 0,28 & 0,62 & 65,0 \\
\hline MD4 & 56,1 & 41,3 & 1,49 & 0,61 & 0,25 & 0,27 & 0,73 & 65,0 \\
\hline
\end{tabular}

\subsection{Avaliação das Misturas através dos Softwares de Termodinâmica Computacional}

Mediante as composições químicas do ferro-gusa e das misturas desfosforantes apresentadas na Erro! Fonte de referência não encontrada. e na Tabela foram realizadas simulações termodinâmicas no FactSage e no ThermoCalc a fim de identificar as variáveis no equilíbrio termodinâmico, quais são a concentração de fósforo de equilíbrio no metal, as fases presentes e quantidade de líquido nas misturas iniciais e escória gerada ao final do processo.

\subsubsection{Caracterização das misturas}

A determinação da quantidade de líquido e fases sólidas formadas nas misturas propostas no presente trabalho foram realizadas tanto através do software FactSage quanto o ThermoCalc.

$\mathrm{Na}$ avaliação através do ThermoCalc, utilizou-se o banco de dados Slag3. Já através do FactSage utilizou-se os bancos de dados Fact53 e FToxid. O Fact53 atua na 
determinação dos compostos sólidos formados e o FToxid permite a avaliação dos compostos formados na fase líquida da mistura/escória.

\subsubsection{Determinação das condições de equilíbrio termodinâmico da reação de desfosforação}

$\mathrm{Na}$ avaliação das condições de equilíbrio termodinâmico da reação de desfosforação utilizou-se os softwares FactSage e ThermoCalc. No caso do FactSage para a determinação das condições de equilíbrio do ferro-gusa, foi aplicado o banco de dados FSstel, e para a determinação das fases formadas na escória gerada no equilíbrio termodinâmico foi utilizado os bancos de dados Fact53 e FToxid. Já para determinação via ThermoCalc o banco de dados utilizado foi o Slag3.

\section{RESULTADOS E DISCUSSÃO}

\subsection{Resultados Experimentais}

A eficiência foi calculado através da equação a seguir.

$$
\eta(\%)=\frac{\left(\left[\% P_{i}\right]-\left[\% P_{f}\right\rfloor\right)}{\left[\% P_{i}\right]} \times 100
$$

Tabela 3. Variação da composição química do ferro-gusa com o tempo nos testes experimentais de desfosforação a $1400^{\circ} \mathrm{C}$.

\begin{tabular}{|c|c|c|c|c|c|c|c|c|}
\hline \multicolumn{2}{|c|}{ Tempo (min) } & 0 & 5 & 10 & 15 & 20 & 30 & $\eta(\%)$ \\
\hline MD1 & $\% P$ & 0,0961 & 0,0652 & 0,0282 & 0,0271 & 0,0259 & 0,0251 & 73,88 \\
\hline MD2 & $\% P$ & 0,0897 & 0,0314 & 0,027 & 0,0254 & 0,023 & 0,0205 & 77,15 \\
\hline MD3 & $\% \mathbf{P}$ & 0,1026 & 0,0277 & 0,0219 & 0,0193 & 0,0166 & 0,0107 & 89,57 \\
\hline MD4 & $\% \mathrm{P}$ & 0,1037 & 0,0263 & 0,0237 & 0,0229 & 0,0218 & 0,0213 & 79,46 \\
\hline
\end{tabular}

Observa-se inicialmente a partir da Tabela 3 que, a redução na concentração do fósforo no ferro-gusa ocorreu de maneira satisfatória, podendo ser concluído preliminarmente que as misturas propostas possuem capacidade desfosforante de reduzir a concentração de tais elementos presentes no ferro-gusa. A Figura 2 mostra a eficiência desfosforante obtido nos testes experimentais.

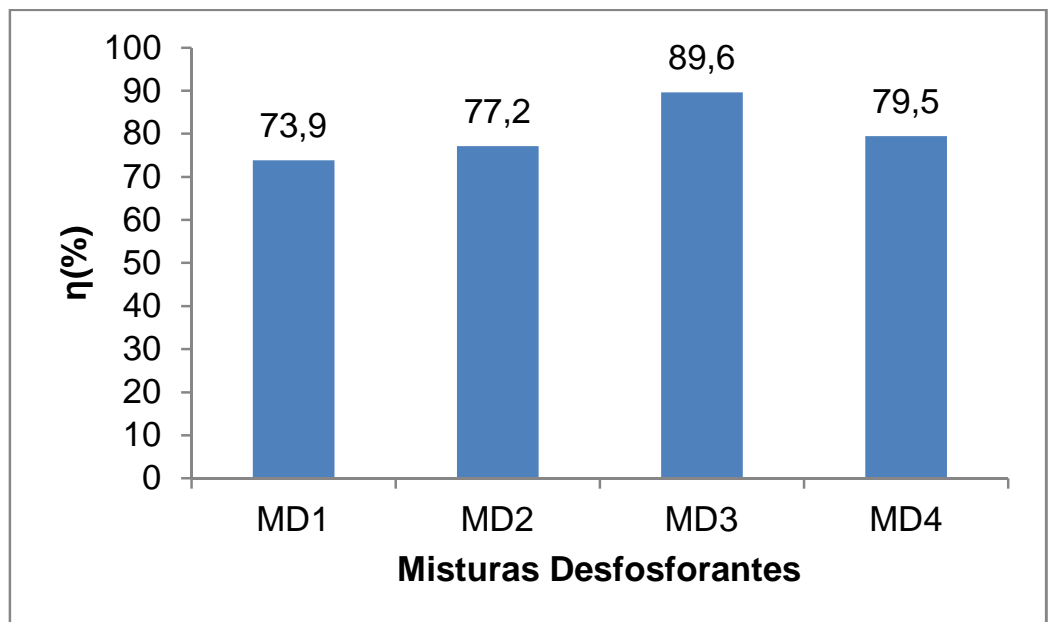

Figura 2. Eficiência Desfosforante (DeP) obtido nos testes experimentais a $1400^{\circ} \mathrm{C}$. 
Observa-se, a partir da Figura 2, que o emprego de todas as misturas desfosforantes propostas no presente trabalho apresenta eficiência desfosforante maior que $70 \%$. Além disso, de acordo com a Figura 2, a mistura MD3 apresentou maior capacidade desfosforante com eficiência de 89,6\%. A mistura MD1 mostrou-se menos eficaz do que as demais no que tange a redução da concentração de fósforo do ferro-gusa, com eficiência de 73,9\%. A Figura $3 a$ mostra a variação da concentração de fósforo com o tempo e a Figura 3b mostra a variação da relação $\left[\% \mathrm{P}_{\mathrm{t}}\right] /\left[\% \mathrm{P}_{0}\right]$ no ferro-gusa mediante o tempo. Onde $\left[\% \mathrm{P}_{\mathrm{t}}\right]$ é a concentração de fósforo no ferro-gusa em um tempo qualquer e $\left[\% \mathrm{P}_{0}\right]$ é a concentração inicial de fósforo no ferro-gusa.

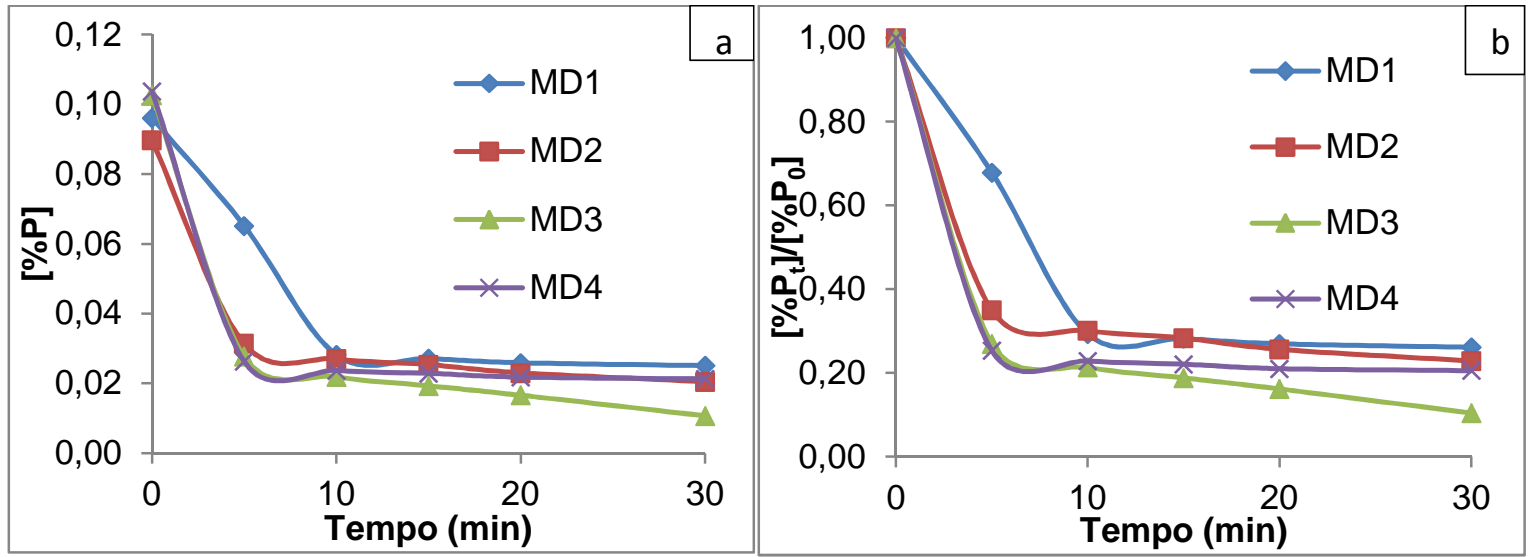

Figura 3. a) Variação da concentração de fósforo [\%P] com o tempo e b) variação da relação $\left[\% \mathrm{P}_{\mathrm{t}}\right] /\left[\% \mathrm{P}_{0}\right]$ no ferro-gusa mediante o tempo nos testes de DeP realizados a $1400^{\circ} \mathrm{C}$.

Observa-se a partir das Figuras $3 a$ e $3 b$ que quando aplicadas as misturas MD2, MD3 e MD4 houve uma redução considerável na concentração de fósforo do ferrogusa nos cinco primeiros minutos de experimento. No entanto, a mistura MD1 não apresentou tal comportamento e, tal fato pode estar atrelado a maior concentração inicial de silício no ferro-gusa quando aplicada esta mistura, conforme mostrado na Tabela 3. É possível notar também a partir das Figuras $3 a$ e $3 b$ que as misturas MD2, MD3 e MD4 apresentam uma redução suave na concentração de fósforo a partir do $10^{\circ}$ minuto de experimento. Já a mistura MD3 mostra-se capaz de reduzir a concentração de fósforo após 30 minutos de experimento, fato esse evidenciado através da inclinação da curva da mistura. A fim de se obter maiores esclarecimentos e conclusões sobre o comportamento das misturas empregadas nos testes experimentais de desfosforação, as mesmas foram avaliadas através dos softwares termodinâmicos. A próxima seção aborda tal avaliação.

\subsection{Avaliação Termodinâmica das Misturas Desfosforantes através dos Softwares de Termodinâmica Computacional}

A Tabela 4 mostra a quantidade de líquido, possíveis compostos sólidos formados e a atividade do $\mathrm{CaO}$ e do FeO obtidas através do ThermoCalc e FactSage. 
Tabela 4. Fases formadas e atividade do $\mathrm{CaO}$ e $\mathrm{FeO}$ na mistura desfosforante a $1400^{\circ} \mathrm{C}$ obtidas a partir do ThermoCalc e FactSage.

\begin{tabular}{|c|c|c|c|c|c|c|c|c|c|c|}
\hline \multirow{3}{*}{$\begin{array}{c}\text { Mistura } \\
\text { MD1 }\end{array}$} & \multirow{3}{*}{$\begin{array}{c}\text { \%Líquido } \\
100,0\end{array}$} & \multicolumn{4}{|c|}{ Fases sólidas formadas } & \multirow{2}{*}{\multicolumn{2}{|c|}{$\mathbf{a}_{\mathrm{CaO}}$}} & \multirow{2}{*}{\multicolumn{2}{|c|}{$a_{\mathrm{FeO}}$}} & \multirow{3}{*}{$\begin{array}{l}n(\%) \\
73,9\end{array}$} \\
\hline & & \multicolumn{2}{|c|}{$\% \mathrm{CaO}$} & \multicolumn{2}{|c|}{$\% \mathrm{MgO}$} & & & & & \\
\hline & & 100,0 & - & - & - & 0,9 & 0,8 & 0,7 & 0,6 & \\
\hline MD2 & 95,3 & 99,3 & 4,7 & 0,7 & - & 1,0 & 1,0 & 0,7 & 0,4 & 77,2 \\
\hline MD3 & 87,2 & 91,8 & 13,0 & 8,2 & - & 1,0 & 1,0 & 0,7 & 0,4 & 89,6 \\
\hline MD4 & 82,0 & 83,3 & 18,0 & 17,0 & - & 1,0 & 1,0 & 0,7 & 0,4 & 79,5 \\
\hline
\end{tabular}

${ }^{*}$ Os valores destacados são referentes aos dados obtidos através do FactSage.

Pode-se notar a partir da Tabela 4 que os dados referentes à quantidade de líquido e fases sólidas formadas nas misturas desfosforantes obtidos através dos softwares ThermoCalc e FactSage são relativamente semelhantes. Observa-se também que com exceção da mistura MD1, que está totalmente líquida a $1400^{\circ} \mathrm{C}$, as demais possuem uma quantidade de fase sólida formada, a qual é constituída somente de $\mathrm{CaO}$. Portanto, preliminarmente, pode-se concluir que dentre as misturas propostas, a mistura MD2 seria a mais eficiente no que tange a remoção do fósforo, uma vez que a mesma apresenta maior quantidade de líquido e possui atividade do $\mathrm{CaO}$ igual a 1. No entanto, no decorrer dos testes experimentais ocorrem reações que acarretam a alteração na composição química da mistura e, tal fato justifica a maior eficiência desfosforante obtido para a mistura MD3. Tendo em vista tal fato, as escórias geradas ao final do processo de desfosforação foram analisadas quimicamente, e em seguida, avaliadas através do FactSage. A Tabela 5 mostra a concentração da escória final obtida nos testes de desfosforação e as Tabelas 6 e 7 mostram as fases formadas e as atividades do $\mathrm{CaO}$ e FeO na escória final obtidas através do FactSage e ThermoCalc, respectivamente.

Tabela 5. Concentração final da escória gerada nos testes de desfosforação a $1400^{\circ} \mathrm{C}$.

\begin{tabular}{cccccccc}
\hline \multirow{2}{*}{ Mistura } & \multicolumn{7}{c}{ Composição (\%) } \\
\cline { 2 - 8 } & $\mathrm{FeO}$ & $\mathrm{CaO}$ & $\mathrm{SiO}_{2}$ & $\mathrm{Al}_{2} \mathbf{O}_{3}$ & $\mathbf{M g O}$ & $\mathrm{MnO}$ & $\mathbf{P}_{2} \mathbf{O}_{5}$ \\
\hline MD1 & 42,40 & 32,30 & 14,70 & 1,40 & 3,91 & 0,60 & 4,69 \\
MD2 & 35,20 & 41,10 & 13,80 & 2,84 & 2,14 & 0,55 & 4,37 \\
MD3 & 27,70 & 52,30 & 11,50 & 2,11 & 1,86 & 0,30 & 4,23 \\
MD4 & 24,40 & 57,10 & 12,20 & 0,90 & 1,17 & 0,30 & 3,93
\end{tabular}

Tabela 6. Fases formadas na escória gerada ao final do processo de desfosforação e atividade do $\mathrm{CaO}$ e $\mathrm{FeO}$ a $1400^{\circ} \mathrm{C}-\mathrm{FactSage}$.

\begin{tabular}{ccccccccc}
\hline \multirow{2}{*}{ Mist. } & \multirow{2}{*}{ \%Líq. } & \multicolumn{2}{c}{ Fases sólidas formadas } & \multirow{2}{*}{$\mathbf{a}_{\mathrm{CaO}}$} & $\mathbf{a}_{\mathrm{FeO}}$ & $\begin{array}{c}\text { Visc. } \\
\text { (P) }\end{array}$ & $\begin{array}{c}\mathbf{n} \\
\mathbf{( \% )}\end{array}$ & Fator \\
\hline MD1 & 93 & 7,0 & - & 0,11 & 0,75 & 0,4 & 73,9 & 1,79 \\
MD2 & 97,5 & 2,5 & - & 0,27 & 0,59 & 0,6 & 77,2 & 1,83 \\
MD3 & 93,8 & - & 6,2 & 1,00 & 0,29 & 1,0 & 89,6 & 2,23 \\
MD4 & 86,7 & - & 13,3 & 1,00 & 0,28 & 1,4 & 79,5 & 2,15 \\
\hline
\end{tabular}


Tabela 7. Fases formadas na escória gerada ao final do processo de desfosforação e atividade do $\mathrm{CaO}$ e $\mathrm{FeO}$ a $1400^{\circ} \mathrm{C}-$ ThermoCalc.

\begin{tabular}{|c|c|c|c|c|c|c|c|c|c|}
\hline \multirow[b]{2}{*}{ Mist. } & \multirow[b]{2}{*}{ \%Liq. } & \multicolumn{3}{|c|}{ Fases sólidas formadas } & \multirow[b]{2}{*}{$\mathbf{a}_{\mathrm{caO}}$} & \multirow[b]{2}{*}{$\mathrm{a}_{\mathrm{FeO}}$} & \multirow{2}{*}{$\begin{array}{c}\text { Visc. } \\
\text { (P) }\end{array}$} & \multirow[b]{2}{*}{$\eta(\%)$} & \multirow[b]{2}{*}{ Fator } \\
\hline & & $\% \mathrm{CaO}$ & $\% 3 \mathrm{CaO} . \mathrm{SiO} 2$ & $\% \mathrm{MgO}$ & & & & & \\
\hline MD1 & 100 & - & - & & 0,17 & 0,78 & 0,4 & 73,9 & 1,95 \\
\hline MD2 & 100 & - & - & - & 0,21 & 0,76 & 0,6 & 77,2 & 1,97 \\
\hline MD3 & 66,2 & 2,8 & 30,5 & 0,4 & 1,00 & 0,51 & 1,0 & 89,6 & 2,17 \\
\hline MD4 & 56,1 & 8,7 & 35,2 & - & 1,00 & 0,53 & 1,4 & 79,5 & 2,09 \\
\hline
\end{tabular}

As Tabelas 6 e 7 mostram que a escória gerada mediante a utilização das misturas MD3 e MD4 estavam saturadas em $\mathrm{CaO}$ ao final do processo de desfosforação, no entanto, a concentração de $\mathrm{CaO}$ sólido na escória MD3 é menor do que a da MD4, com $6,2 \%$ e $13,3 \%$ (FactSage) e 2,8\% e 8,7\% (ThermoCalc), respectivamente. Já as escórias MD1 e MD2 não estavam saturadas em $\mathrm{CaO}$ ao final do processo de desfosforação, no entanto, as mesmas apresentaram a formação do $3 \mathrm{CaO} \cdot \mathrm{P}_{2} \mathrm{O}_{5}$ sólido (FactSage), enquanto o ThermoCalc apresentou que ambas as misturas estão totalmente liquidas na temperatura de trabalho. Através da Tabela 6 pode-se observar também que as escórias mais eficientes foram aquelas que, ao final do processo de desfosforação, possuíam atividade do $\mathrm{CaO}$ igual a 1 e não maior quantidade de líquido. Porém, entre as escórias saturadas em $\mathrm{CaO}$ (MD3 e MD4), àquela com maior quantidade de líquido (MD3) e maior atividade do $\mathrm{FeO}$ foi mais eficiente. Tal comportamento ocorreu devido à mudança de composição química que ocorreu nas misturas no decorrer da reação de desfosforação.

Pra analisar a influencia conjunta destes parâmetros foi criado o fator de Desfosforação (F) de acordo com a equação (8)

$$
\text { Fator de } \operatorname{DeP}(F)=a_{C a O}+a_{F e O}+\left(\% \frac{L i q}{100}\right)
$$

A Figura 4a e Figura 4b mostram a correlação entre o Fator de DeP, qual leva em conta a $\mathrm{a}_{\mathrm{CaO}}, \mathrm{a}_{\mathrm{FeO}} \mathrm{e} \%$ Liquido, e a eficiência das misturas.
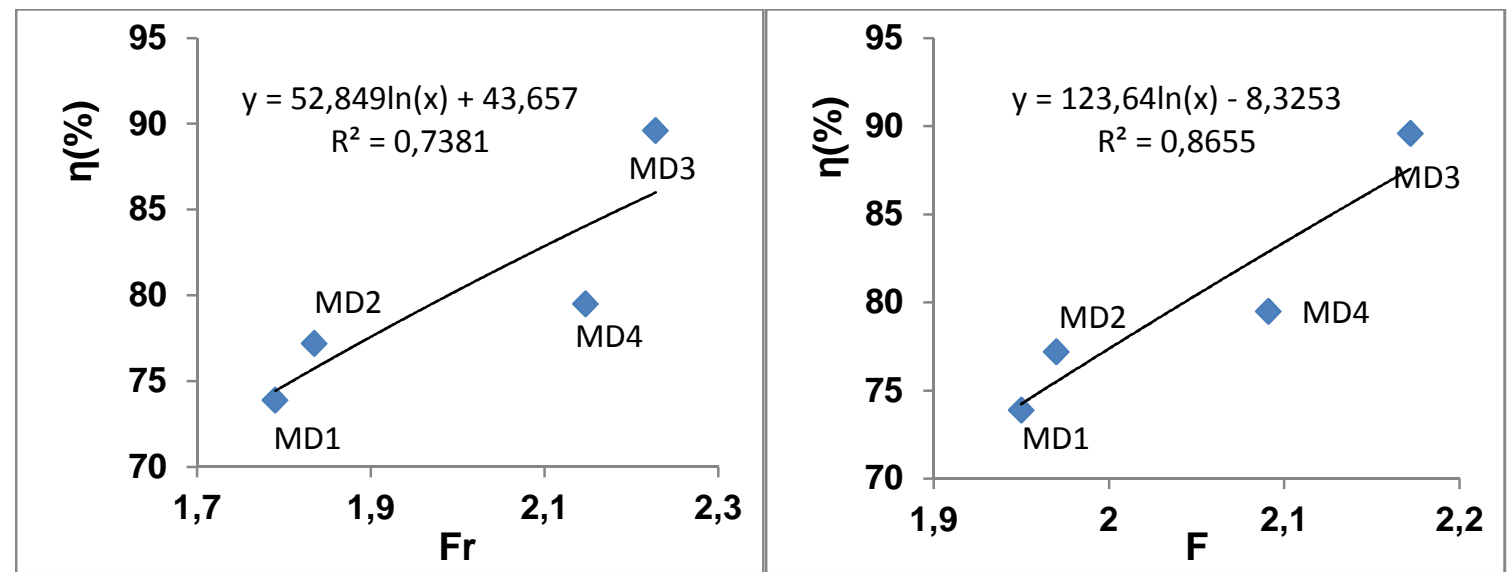

Figura 4. a) Fator $x$ Eficiência Desfosforante (DeP) - FactSage e b) Fator x Eficiência Desfosforante (DeP) - ThermoCalc.

Como pode-se ver na Figura 4, a correlação $\left(R^{2}\right)$ entre $F$ e a eficiência de DeP foram de 0,747 e 0,872 pelo FactSage e ThermoCalc, respectivamente. Como este valor pode ser considerado alto, pode-se então criar um modelo para prever $\eta$ usando a equação da linha de tendência gerada na Figura 4. Como obteve-se uma correlação maior usando o Thermocalc, será usada a equação gerada nestes caso, mostrada nas equações (9) e (10).

$$
y=123,64 \ln (x)-8,3253
$$


O comportamento da mistura inicial, da escória final e do processo de desfosforação abordado no presente trabalho podem ser explicados através da Figura e da Tabela 8.

Tabela 8. Variação instantânea da força motriz, de $k$ e da velocidade da reação mediante emprego das misturas desfosforantes a $1400^{\circ} \mathrm{C}$.

\begin{tabular}{|c|c|c|c|c|c|c|c|}
\hline & рро (min) & 0 & 5 & 10 & 15 & 20 & 30 \\
\hline \multirow{4}{*}{ MD1 } & {$[\% \mathrm{Pi}]$} & 0,0961 & 0,0652 & 0,0282 & 0,0271 & 0,0259 & 0,0251 \\
\hline & $\% P$ - \%Peq & 0,096 & 0,0651 & 0,0281 & 0,027 & 0,0258 & 0,025 \\
\hline & $k\left(\min ^{-1}\right)$ & 0 & 0,0776 & 0,168 & 0,0079 & 0,0091 & 0,0031 \\
\hline & $\mathrm{d}[\% \mathrm{P}] / \mathrm{dt}$ & 0 & $5,06.10^{-3}$ & $4,72.10^{-3}$ & $2,16.10^{-4}$ & $2,35.10^{-4}$ & $7,87.10^{-5}$ \\
\hline \multirow{4}{*}{ MD2 } & [\%Pi] & 0,0897 & 0,0314 & 0,027 & 0,0254 & 0,023 & 0,0205 \\
\hline & \%P - \%Peq & 0,0897 & 0,0314 & 0,027 & 0,0254 & 0,023 & 0,0205 \\
\hline & $k\left(\min ^{-1}\right)$ & 0 & 0,21 & 0,0302 & 0,0122 & 0,0198 & 0,0115 \\
\hline & $\mathrm{d}[\% \mathrm{P}] / \mathrm{dt}$ & 0 & $6,59 \cdot 10^{-3}$ & $8,15 \cdot 10^{-4}$ & $3,10 \cdot 10^{-4}$ & $4,57.10^{-4}$ & $2,36 \cdot 10^{-5}$ \\
\hline \multirow{4}{*}{ MD3 } & [\%Pi] & 0,1026 & 0,0277 & 0,0219 & 0,0193 & 0,0166 & 0,0107 \\
\hline & \%P - \%Peq & 0,1026 & 0,0277 & 0,0219 & 0,0193 & 0,0166 & 0,0107 \\
\hline & $k\left(\min ^{-1}\right)$ & 0 & 0,2619 & 0,047 & 0,0253 & 0,0302 & 0,0439 \\
\hline & $\mathrm{d}[\% \mathrm{P}] / \mathrm{dt}$ & 0 & $7,25.10^{-3}$ & $1,03 \cdot 10^{-3}$ & $4,88 \cdot 10^{-4}$ & $5,00 \cdot 10^{-4}$ & $4,70.10^{-4}$ \\
\hline \multirow{4}{*}{ MD4 } & [\%Pi] & 0,1037 & 0,0263 & 0,0237 & 0,0229 & 0,0218 & 0,0213 \\
\hline & $\% \mathrm{P}-\%$ Peq & 0,1037 & 0,0263 & 0,0237 & 0,0229 & 0,0218 & 0,0213 \\
\hline & $k\left(\min ^{-1}\right)$ & 0 & 0,2745 & 0,0208 & 0,0068 & 0,0098 & 0,0023 \\
\hline & $\mathrm{d}[\% \mathrm{P}] / \mathrm{dt}$ & 0 & $7,21 \cdot 10^{-3}$ & $4,93 \cdot 10^{-4}$ & $1,57.10^{-4}$ & $2,15.10^{-4}$ & $4,94 \cdot 10^{-5}$ \\
\hline
\end{tabular}

Através da análise da Figura 3 nota-se que nos primeiros 5 minutos de experimento, as misturas com atividade do $\mathrm{CaO}$ igual a 1 (MD2, MD3 e MD4) foram as que tiveram maior velocidade de reação e, consequentemente, menores concentrações de fósforo ao mesmo tempo que a mistura MD1, a qual não estava saturada em $\mathrm{CaO}$ inicialmente. No entanto, à medida que as reações ocorreram, as escórias tiveram alteração em sua composição química e, a partir deste momento, a atividade do $\mathrm{CaO}$ na escória MD2 não era mais 1, acarretando a diminuição da sua velocidade.

Já as escórias MD3 e MD4 apresentaram a mesma velocidade de reação até o tempo de 10 minutos e, a partir daí, a velocidade de reação da escória MD3 foi maior. Tal comportamento aconteceu devido a maior quantidade de líquido da mistura MD3 com relação a MD4. Além disso, a Tabela 8 mostra que, em todos os casos, os valores da força motriz seguiram a tendência da concentração inicial de fósforo no ferro-gusa, o qual está atrelado à baixa concentração de fósforo de equilíbrio. Portanto, com base no exposto, poder-se-ia concluir erroneamente que as maiores eficiências obtidas nos testes experimentais foram simplesmente devido à maiores concentrações de fósforo inicial do ferro-gusa utilizado. No entanto, não é possível fazer tal afirmação, uma vez que as misturas que obtiveram maior eficiência também apresentaram maiores valores de $\mathrm{k}$ e menores concentrações finais de fósforo, ou seja, as misturas passaram por alterações em suas composições químicas, as quais permitiram o alcance do maior eficiência.

Observa-se ainda através da Tabela 8 que as misturas que apresentaram atividade do $\mathrm{CaO}$ igual a 1, apresentaram também maiores valores de $\mathrm{k}$. Além disso, 0 aumento na velocidade da mistura MD2 com relação a MD4 após 10 minutos de experimento foi devido ao aumento do valor de $\mathrm{k}$ nesse intervalo de tempo. Neste 
caso, como $\mathrm{k}$ depende da quantidade de líquido na mistura, pode-se inferir que a mistura MD4 apresentava menor quantidade de líquido com relação a mistura MD2.

Portanto, pode-se afirmar com base nos resultados encontrados no presente trabalho que as escórias que tiveram atividade do $\mathrm{CaO}$ igual a 1 apresentaram melhores desempenhos desde que as mesmas apresentassem a quantidade de líquido superior a $83 \%$. A atividade do $\mathrm{CaO}$, além de influenciar na concentração de fósforo de equilíbrio, também possui influência sobre o coeficiente de atividade do $\mathrm{P}_{2} \mathrm{O}_{5}$ e, consequentemente, na concentração de $\mathrm{P}_{2} \mathrm{O}_{5}$ na escória.

Além disso, sabe-se que o valor de k está relacionado ao transporte de massa do fósforo no metal e na escória, como mostrado na Equação 3. Assim, maiores concentrações de $\mathrm{CaO}$ acarretam a diminuição do coeficiente de atividade do $\mathrm{P}_{2} \mathrm{O}_{5}$ permitindo maiores concentrações de equilíbrio de $\mathrm{P}_{2} \mathrm{O}_{5}$ na escória. Desta maneira, se admitirmos que a velocidade da reação de desfosforação é controlada pelo trasporte de massa na escória, conforme abordado na Equação 4, um aumento na concentração de fósforo de equilíbrio na escória acarreta o aumento na velocidade de desfosforação. Por fim, conclui-se que uma análise das misturas iniciais através do FactSage ou ThermoCalc não é suficiente para se determinar a mistura que será mais eficiente, ou seja, é necessário analisar as escórias finais e aquela que apresentar atividade do $\mathrm{CaO}$ igual a 1 e maior quantidade de líquido na situação final será a mais eficiente.

Pode-se afirmar também que através da analise através da termodinâmica computacional das escórias finais, a mistura que possuir atividade do $\mathrm{CaO}$ igual a 1 apresentam maior quantidade de liquido e atividade do FeO será a mais eficiente. Porém a quantidade de liquido e a atividade do $\mathrm{FeO}$ devem ser as maiores possíveis, desde que não diminua a atividade do $\mathrm{CaO}$.

\section{CONCLUSÃo}

Diante dos resultados obtidos neste trabalho pode-se concluir que:

- Os testes experimentais mostraram que a reação de desfosforação ocorreu de maneira efetiva até o $5^{\circ}$ minuto de experimento e, a partir do $10^{\circ}$ minuto, a redução na concentração de fósforo ocorreu de maneira suave;

- A comparação entre os resultados experimentais e os parâmetros termodinâmicos permitiu identificar que o aumento da basicidade ótica, da capacidade de fosfato e do coeficiente de partição do fósforo acarreta um aumento na eficiência desfosforante das misturas. Tal eficiência também é favorecida quanto menor for 0 coeficiente de atividade do $\mathrm{P}_{2} \mathrm{O}_{5}$ na escória;

- Os softwares ThermoCalc e FactSage mostraram que o aumento da concentração de $\mathrm{CaO}$ na mistura contribui para o aumento da temperatura de fusão da mesma e, consequentemente, diminuição da quantidade de líquido formado;

- A presença de $\mathrm{CaO}$ nas misturas desfosforantes contribui para a fixação do $\mathrm{P}_{2} \mathrm{O}_{5}$ na escória e, à medida que se aumenta a concentração do $\mathrm{CaO}$, o composto $3 \mathrm{CaO} \cdot \mathrm{P}_{2} \mathrm{O}_{5}$ é fixado na escória líquida. Tal fato foi comprovado a partir da situação de equilíbrio gerada pelo software ThermoCalc e FactSage;

- Através do FactSage foi possível identificar que o aumento da atividade do $\mathrm{CaO}$ e a diminuição da atividade do FeO favorecem o aumento da eficiência desfosforante; Por fim, após confrontar os resultados experimentais e àquelas obtidos a partir dos softwares de termodinâmica computacional conclui-se que uma análise das misturas iniciais através do FactSage / ThermoCalc não é suficiente para se determinar a mistura que será mais eficiente, ou seja, é necessário analisar as escórias finais e 
àquela que apresentar atividade do $\mathrm{CaO}$ igual a 1 e maior quantidade de líquido e atividade do $\mathrm{FeO}$ na situação final será a mais eficiente.

- O Modelo para prever a eficiência de DeP nas condições usadas no presente trabalho é: $\eta(\%)=123,64 \ln (F)-8,3253$

\section{Agradecimentos}

A CAPES pela bolsa de mestrado.

\section{REFERÊNCIAS}

1 MUKHERJEE, T.; CHATTERJEE, A. Production of low phosphorus steels from high phosphorus Indian hot metal: Experience at Tata Steel. Bulletin of Material Science, $\mathrm{n}$. 6, v. 19, p. 893-903, 1996.

2 CAMPOS, V. F. Curso sobre Pré-refino de Ferro-Gusa. 1. ed. Belo Horizonte: Universidade Federal de Minas Gerais, 1984. 31p.

3 TURKDOGAN, E.T. Fundamentals of Steelmaking. 1. ed. London: The Institute of Materials, 1996. 331p.

4 HEALY, G. A New Look at Phosphorus Distribution. Journal of the Iron and Steel Institute, n. 6, v. 208, p. 664-668, 1970.

5 WEI, P.; SANO, M.; HIRASAWA, M.; MORI, K. Kinects of Phosphorus Transfer between Iron Oxide Containing Slag and Molten Iron of High Carbon Concentration under Ar-O2 Atmosphere. ISIJ International, n. 4, v. 33, p. 479-487, 1993. 